This item was submitted to Loughborough's Research Repository by the author.

Items in Figshare are protected by copyright, with all rights reserved, unless otherwise indicated.

\title{
Automated engine calibration validation for real world driving conditions
}

PLEASE CITE THE PUBLISHED VERSION

https://doi.org/10.1504/IJPT.2020.111251

PUBLISHER

Inderscience

VERSION

AM (Accepted Manuscript)

PUBLISHER STATEMENT

This paper was accepted for publication in the journal International Journal of Powertrains and the definitive published version is available at https://doi.org/10.1504/IJPT.2020.111251

\section{LICENCE}

CC BY-NC-ND 4.0

\section{REPOSITORY RECORD}

Fletcher, Tom, Nikolaos Kalantzis, Mark Cary, Bob Lygoe, Antonios Pezouvanis, and Kambiz Ebrahimi. 2020. "Automated Engine Calibration Validation for Real World Driving Conditions". Loughborough University. https://hdl.handle.net/2134/12951578.v1. 


\title{
Automated Engine Calibration Validation for Real World Driving Conditions
}

\author{
Tom Fletcher ${ }^{1}$, Nikolaos Kalantzis ${ }^{1}$, Mark Cary ${ }^{2}$, Bob Lygoe ${ }^{2}$, Antonios Pezouvanis ${ }^{1}$, \\ Kambiz Ebrahimi $^{1}$
}

\begin{abstract}
With the introduction of the Worldwide Harmonised Light Vehicles Test Procedure (WLTP) and Real Driving Emissions (RDE) legislative tests, it has become imperative for automotive manufacturers to verify that their vehicles operate reliably and efficiently across a wide range of operating conditions. Engine air charge control and torque delivery should be consistent, smooth, and predictable to the operator, despite disturbances induced by technologies designed to improve efficiency and reduce emissions such as Variable Cam Timing (VCT), Exhaust Gas Recirculation (EGR) and forced induction. This is of particular importance to hybrid and Advanced DriverAssistance Systems (ADAS)-enabled vehicles which require accurate control of the engine torque. The torque strategy validation is an iterative process which can be very time-consuming, expensive and requires considerable experience in order to adjust the calibration to meet validation requirements whilst maintaining accuracy with regards to the original characterisation data. This paper describes development and introduction of an automated validation process at the Ford's Dunton Technical Centre in the UK. The algorithm was developed and tested in conjunction with a co-simulation environment, and both simulated and experimental results are presented. The automated procedure has been shown to reduce validation process time by around $50 \%$ resulting in significant cost saving whilst improving the overall accuracy of the calibration by around $30-50 \%$ depending on the engine programme.
\end{abstract}

\section{Index Terms}

Air Charge Estimation; Model Based Calibration; Co-Simulation; Process Automation; Torque Prediction

\section{INTRODUCTION}

Engine calibration validation (the final "sign-off" of an engine Powertrain Control Module (PCM) calibration) is a crucial step in the development of the new engine variant because it is the last chance for engineers to identify and rectify discrepancies between the predicted engine behaviour and actual engine performance as seen by consumers. It is also an incredibly complex task due to the sheer number of parameters and control actuators used on modern vehicles to meet increasingly stringent emissions regulations [1], [2]. At the same time, international legislators are moving away from testing using modal drive cycles such as the New European Driving Cycle (NEDC) and towards highly dynamic test cycles such as the WLTP and the RDE test in order to bridge the gap between official fuel economy and emissions figures and real-world consumer experience [3]. The combination of these factors is requiring automotive Original Equipment Manufacturers (OEMs) to not only produce highly efficient engines, but also for them to ensure that they operate efficiently at a wide range of operating points rather than just at specific "mapping" points.

This work is part of the Validation Platform for Engine Calibration (VPEC) project funded by the Digital Engineering and Test Centre (DETC) which focusses on the process improvement of the validation of an engine calibration at Ford Motor Company. The validation procedure begins with a completed engine mapping calibration and involves testing the engine on a dynamometer through a series of around 300 validation test points. Once the testing is complete, any error states are identified, and the calibration

\footnotetext{
${ }^{1}$ Loughborough University, Loughborough, UK

${ }^{2}$ Ford Motor Company, Dunton Technical Centre, UK
} 
is adjusted to minimise the error before re-testing. This iterative process often takes several weeks from beginning to end and is performed for every unique engine and vehicle variant before going to production.

One of the aims of this project is to produce an automated calibration process which can be used to optimise the calibration online during dynamometer testing. This will reduce the number of experimental iterations and significantly reduce the cost and time of the validation process. Automation of the validation procedure also enables 24-hour testing, speeding up the process significantly, as well as reducing the workload on calibration Subject Matter Experts (SMEs).

\section{BACKGROUND}

With an internal combustion engine, the purpose of the torque strategy is to determine the throttle and waste gate actuator settings to deliver the necessary airflow that satisfies the desired engine power demand [4]. In addition, the torque delivery needs to be smooth, consistent, and predictable to the operator.

In modern vehicles, the accurate estimation and control of the brake torque output of an engine is becoming increasingly significant for two main reasons. Firstly, progressively stricter emissions regulations are putting more emphasis on precise engine control, especially for hybrid vehicle architectures; which often require the acceleration demand from the driver to be divided between the engine and one or more electric motors [5]-[7], the uptake of ADAS and autonomous vehicles are gradually removing the driver from the control loop. Accurate and reliable estimates of the engine torque are required for ADAS, such as for adaptive cruise control [8], shift quality control [9] and engine speed control [10].

In laboratory settings, the engine indicated torque can be effectively measured by the use of in-cylinder pressure transducers, however, these sensors are prohibitively expensive for consumer vehicle applications and are not reliable enough for production vehicles. Hence it is necessary to estimate the torque indirectly based on the readings from other sensors [11]. Torque estimation is an incredibly complicated task due to the considerable number of design, environmental and control parameters that impact the engine performance. Modern engines have a variety of controllable parameters which will each affect the torque of the engine, ranging from the basics such as throttle position, ignition timing and fuel injection timing to more innovative technologies such as VCT, EGR and multiple-stage turbocharging [12], [13].

Furthermore, many torque error states are a function of the coupling between the strategy air charge estimation feature and torque. Typically, representations of the engine indicated efficiency, optimal and borderline spark settings, and so on, used for torque inference are stored in Look Up Tables (LUTs). These are usually indexed using (normalised) trapped air charge. Therefore, errors in air charge estimation directly influence the output from the associated LUTs, distorting the corresponding strategy torque calculations. Speed-density air charge estimators [14]-[19] are particularly sensitive to changes in intake and exhaust hardware. Consequently, for a given engine, a torque calibration which functions adequately in one vehicle application may not function as well in another if the air charge calibration error pattern degrades among applications

There are a number of different approaches to the torque estimation problem. The most basic of these is a fully "mapped" calibration which uses a series of LUTs based on engine speed, mass air flow, spark advance, injected fuel mass, etc., which then need to be populated through extensive testing [20]. This is a highly reliable and deterministic method but suffers from the "curse of dimensionality" as the number of tables increases to include corrections for camshaft timing, EGR, etc.. As a result, the time required to populate the LUTs and the memory required to store them becomes prohibitive for production engines. On the other hand, there are various more advanced indirect measurement techniques such as; sliding mode estimation [21], Kalmann filtering [22]-[24], Unknown Input Observers (UIO) [11], [25], adaptive parameter estimation [11], [25] and dirty differentiation estimation [11], [26]. These techniques use the measurement of other engine and vehicle states such as crankshaft speed and manifold pressure to estimate the brake torque in real-time. 


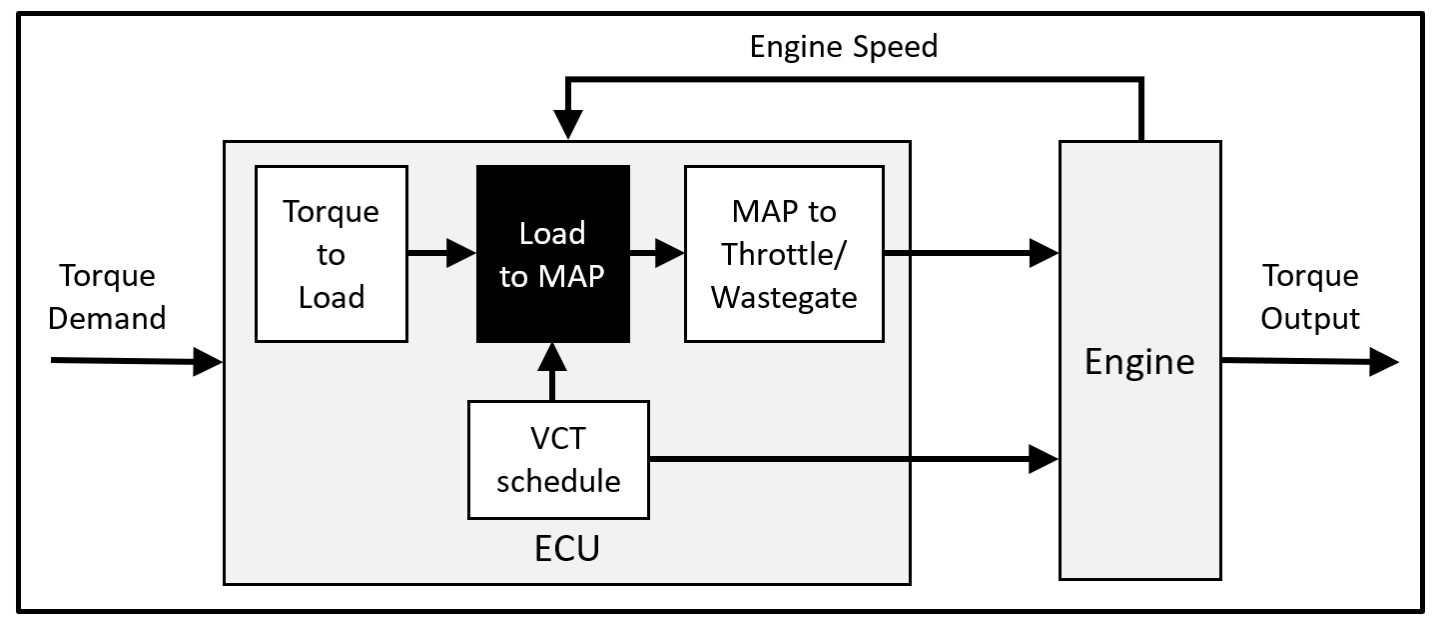

Fig. 1: Ford Gasoline Engine Control (FGEC) Strategy

\section{Ford Gasoline Engine Control (FGEC) Strategy}

The Ford Motor Company uses a semi-empirical model for predictive control of both the air flow and the torque produced by the engine. This technique sits somewhere in between the two categories above, using a number of theoretical assumptions to minimise the requirement for LUTs. This model is calibrated using empirical data gathered at specific "mapping points" represented by variables such as engine speed, manifold pressure and VCT indices. During normal operation, the calibration is then interpolated based on the current state of the engine and the demanded engine torque. This technique balances the requirement for a predictable deterministic response suitable for robust torque control of the engine whilst limiting the requirement for extensive calibration testing. Despite this, the strategy still requires extensive calibration and validation testing to be performed to ensure reliability under real world driving conditions. As a result, it is vital to ensure that testing is performed as efficiently as possible. A simplified version of the torque control strategy used by Ford is outlined in Figure 1. The strategy works as follows;

1) A torque demand is received by the PCM

2) The torque demand is converted to a normalised cylinder air charge (or "load") demand based on LUTs.

3) Simultaneously, the engine speed and torque demand are used to determine the optimum VCT angles based on a predetermined schedule.

4) The VCT angles, engine speed and target load are used to calculate a target Manifold Absolute Pressure (MAP) based on a semi-empirical model.

5) The MAP target is used to control the throttle angle and turbocharger waste-gate using a combination of feed-forward and feedback control.

6) (The engine responds to the control actuations and produces an amount of brake torque)

\section{VALIDATION PROCESS}

Validation is the final step in the development of a production PCM calibration. It involves running the engine using a set of 200-300 independent validation test points (referred to as "test points") to ensure that the interpolation of the mapped LUT breakpoints ("mapping points") defined in the characterisation phase are accurate during real-world driving scenarios.

The most significant difference between the characterisation phase and the validation phase is in the definition of the engine operating point for the test. During the characterisation phase, internal PCM variables are used to manually override the FGEC strategy. This enables accurate control of the independent 
variable in the respective LUT while measurements are taken of the dependent variable. For example, to populate the LUT from MAP to load (normalised air charge), the operating point is defined by the MAP and the load is measured. The semi-empirical models are then calibrated to minimise the error from these measurements (see Figure 2a).

In comparison, during the validation phase the PCM strategy works as it would in the real world without internal PCM variables being overridden. In the case of load control (Step 4 from Section III), the FGEC strategy uses the LUT to convert from a target load to a target MAP. This means that during a validation test, the operating point of the engine is defined by the target load rather than the MAP. The PCM subsequently interpolates the calibration to give a target MAP, which ultimately results in an air flow response from the engine. The measured load is then compared to the desired load and the error is calculated, see Figure 2b. If the error is not acceptable, the calibration is modified. However, the modified calibration will now result in a different MAP for the same target load, meaning that the operating point of the engine will have changed causing changes to other control actuators such as the VCT angles. As a result, all test points affected need to be re-validated on the dynamometer.

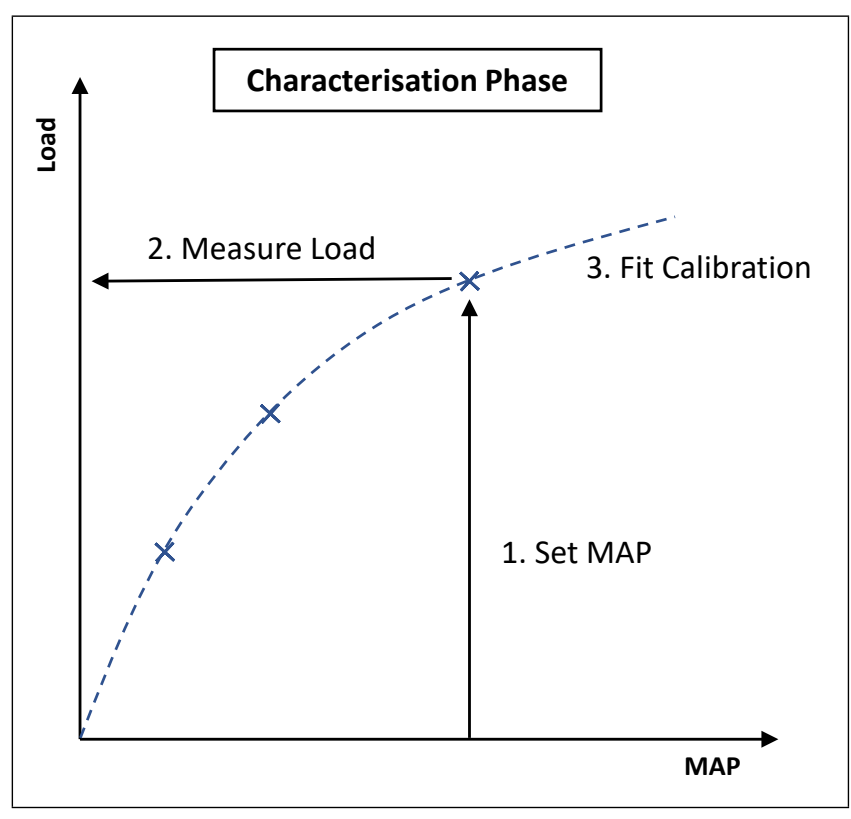

(a) Characterisation Phase

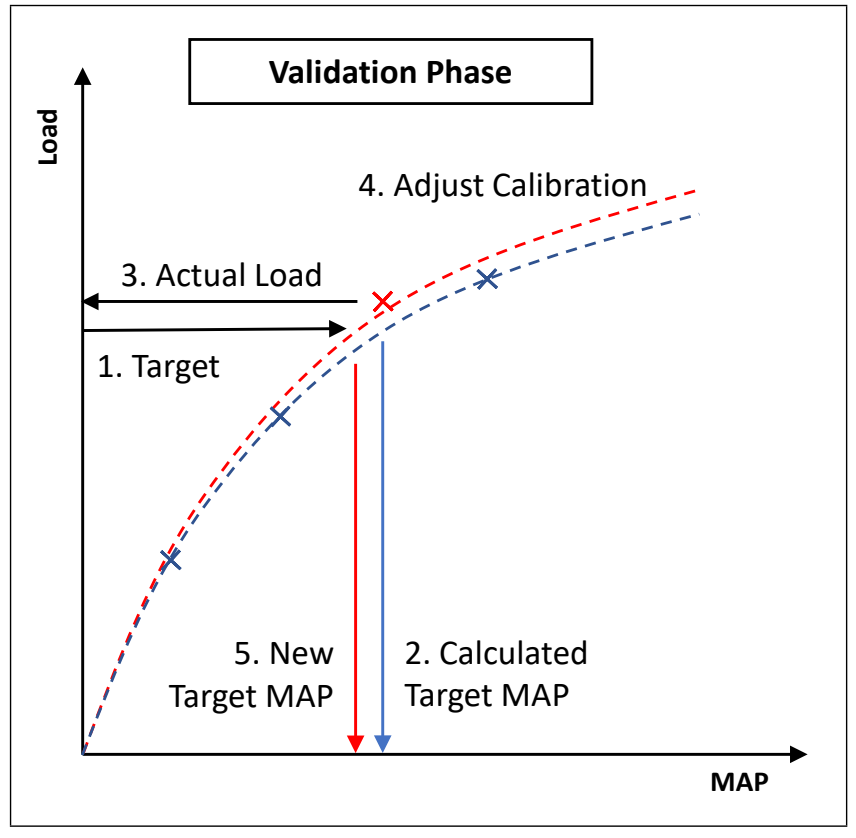

(b) Validation Phase

Fig. 2: Comparison of calibration model fitting during the characterisation and validation phases

The overall process is shown in Figure 3 and described below;

1) An engine base map and PCM calibration is obtained alongside the base map validation data (around 7500 "mapping points") during the characterisation phase.

2) The engine is installed on the dynamometer and the PCM is programmed as closely as possible to how it would be set up in a real vehicle. This means all control actuators are in fully automatic mode.

3) The test cell is used to run the engine through a set of (around 300) pre-determined speed and torque "test points" which represent the full engine operating range at stabilised engine operating temperatures.

a) The demand torque and speed are logged alongside indicator and diagnostic parameters such as the MAP, Mass Air Flow (MAF), and measured brake torque.

4) The logged data is subsequently analysed by SMEs and calibration parameters adjusted to in an attempt to remove error states, while simultaneously also attempting to keep the original base map performance within the error tolerances with respect to the original calibration data. 


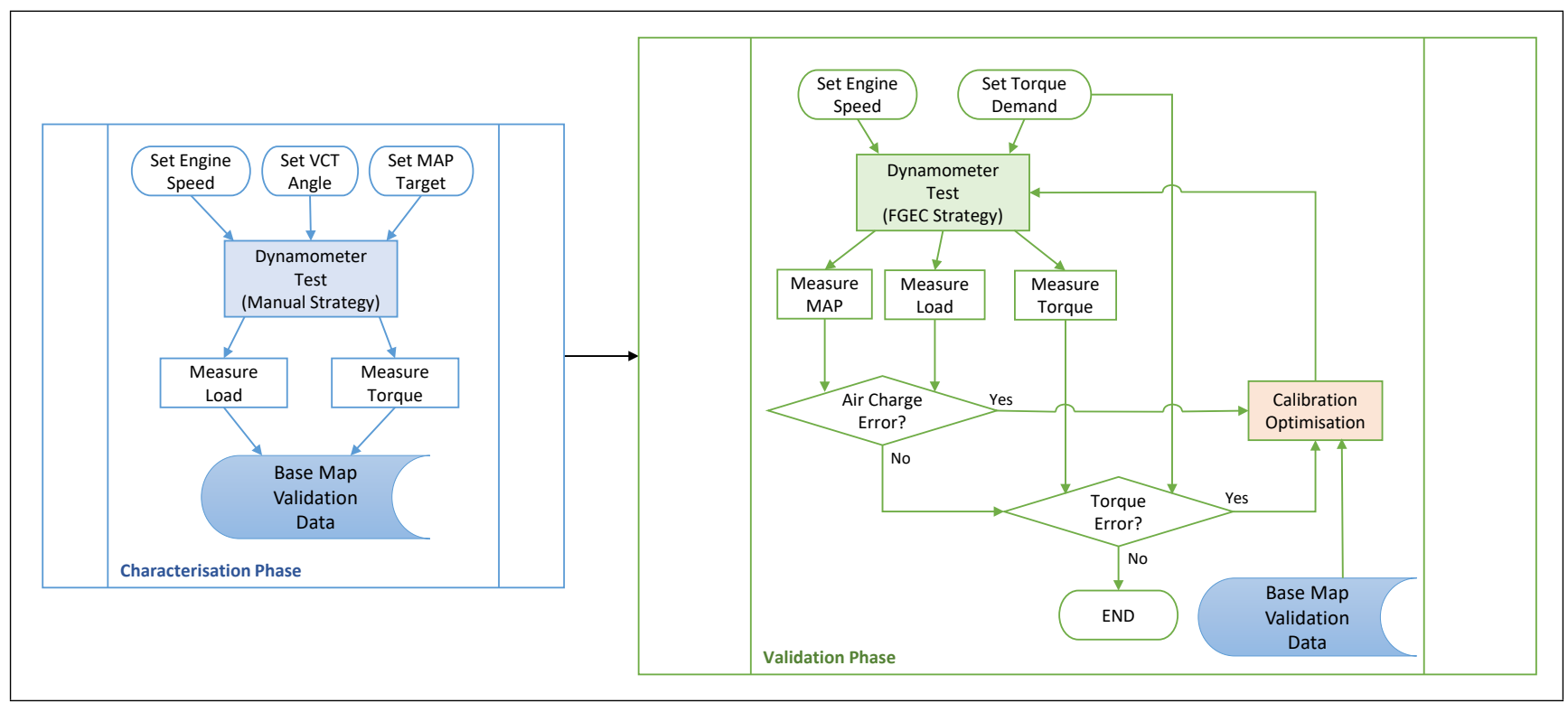

Fig. 3: Characterisation and Validation Process Overview

5) The new calibration is re-tested on the engine dynamometer. Only test points affected by the calibration change are re-examined.

6) Steps 3-5 are repeated until the number of error states has been minimised.

This difference means that the validation effectively checks the accuracy of the calculated response from the strategy, however it introduces an additional layer of complexity. If the calibration does not meet the desired Measures of Success (MoS) at one of the test points (referred to as an "error state"), the calibration may need to be adjusted at a number of the mapping points. Each of these changes will not only affect the operating point of the error state, but also other test points for which these calibration breakpoints points were used. As a result, all affected test points will need to be re-examined.

This intricacy means the validation can be very time consuming, requiring several iterations of testing on the dynamometer and manual adjustment of the calibration. The issue is compounded by the fact that the adjustment of the calibration needs to be performed very carefully to ensure that not only are the validation error states minimised, but also the original calibration data remains within the MoS. Consequently, experienced SMEs are required to perform this task and inconsistent approaches, depending on experience, can affect the reliability of the process. Ultimately, it can be several weeks before an acceptable result is obtained. However, the problem is well suited to computational optimisation techniques which are able to analyse large volumes of data accurately, reliably and in a much shorter timescale than a human.

\section{OPTIMISATION TOOL}

Recall that the controller LUTs are estimated during the characterisation phase using results from rigorous testing at pre-defined mapping points. It assumed that the initial calibration has acceptable errors for all of the original mapping data. However, during normal driving the engine will operate at conditions between the mapping points. It is imperative the calibration returns accurate inferences under these conditions. Evaluating the calibration between operating points requires interpolating the evaluation of individual LUTs. While the calibration may be accurate at the tested operating points there is no guarantee that the interpolated results will be accurate. Consequently, between 2 and 4 LUT values may need to be adjusted to tune the calibration for operation between test operating points in order to eliminate an error state, see Figure 4. 


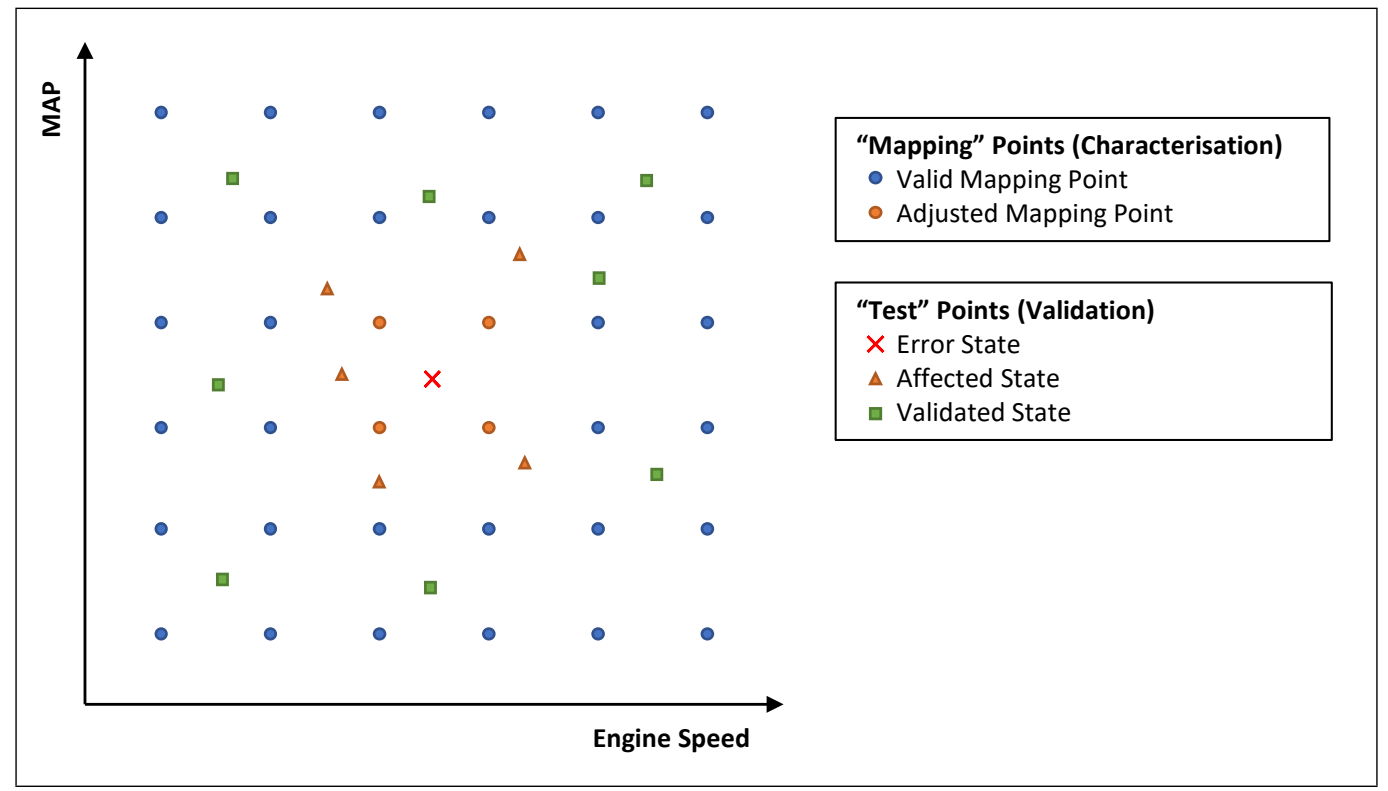

Fig. 4: Requirement for iterative dynamometer testing. Correction of a single error state will affect the calibration at a number of "mapping points". Each adjusted "mapping point" may in-turn affect the operating point of a number of "test points" which will each need to be re-tested.

This task is too often complicated to be undertaken by manually by an engineer. Calibration parameters affect performance at many operating points. Therefore, in fixing one error state it is entirely possible to introduce a new one at a point where previously the calibration performed accurately. Hence, while correcting for errors between test operating points, any changes in error magnitudes at test operating points where the performance was previously acceptable need to be reassessed.

Typically, there are between 5-10 out of 300 validation data points that don't meet the target validation. A test at a single operating point may consist of a few hundred observations with up to 3200 operating points being tested. This is a very large optimisation problem that needs to be reduced before it can be successfully executed. The reduction is performed by identifying all operating points associated with large validation errors and reducing the validation data and original data to operating points of interest. The optimisation problem can still consist of over 10,000 constraints and 1000 LUT values to adjust which is a challenge for standard optimisation routines. A further refinement to the optimisation process was included to start with a series of smaller optimisations that will move the LUT values towards the final solution.

The interior-point non-linear optimiser in the Optimization Toolbox ${ }^{\mathrm{TM}}$ by MathWorks can be used to solve optimisation problems of this size. It uses a combination of direct and conjugate gradient steps to gradually approach the optimal solution by traversing the feasible region, beginning with the feasible, but not necessarily optimal base calibration. It does not directly interact with the dynamometer during the optimisation, however, some routines that take account of the structure of the problem have been developed to efficiently calculate numerical derivatives. This means that once the optimisation is complete, the new calibration requires to be retested at any point which has been affected by the calibration change.

When run to completion, the optimisation routine may take several thousand iterations and several hours of computation time. However, for practical purposes the optimisation can be stopped as soon as all the errors are within the target values, and during trials the optimisation routines have been observed to take between 10 and 180 minutes depending on the size of validation and base data sets. In the event of non-convergence or convergence outside the desired $\mathrm{MoS}$, the optimisation is also limited with respect to the number of iterations and total solve time. Once the optimisation routine is complete, whether by meeting the MoS or due to reaching the maximum number iterations or solve time, the new calibration is 


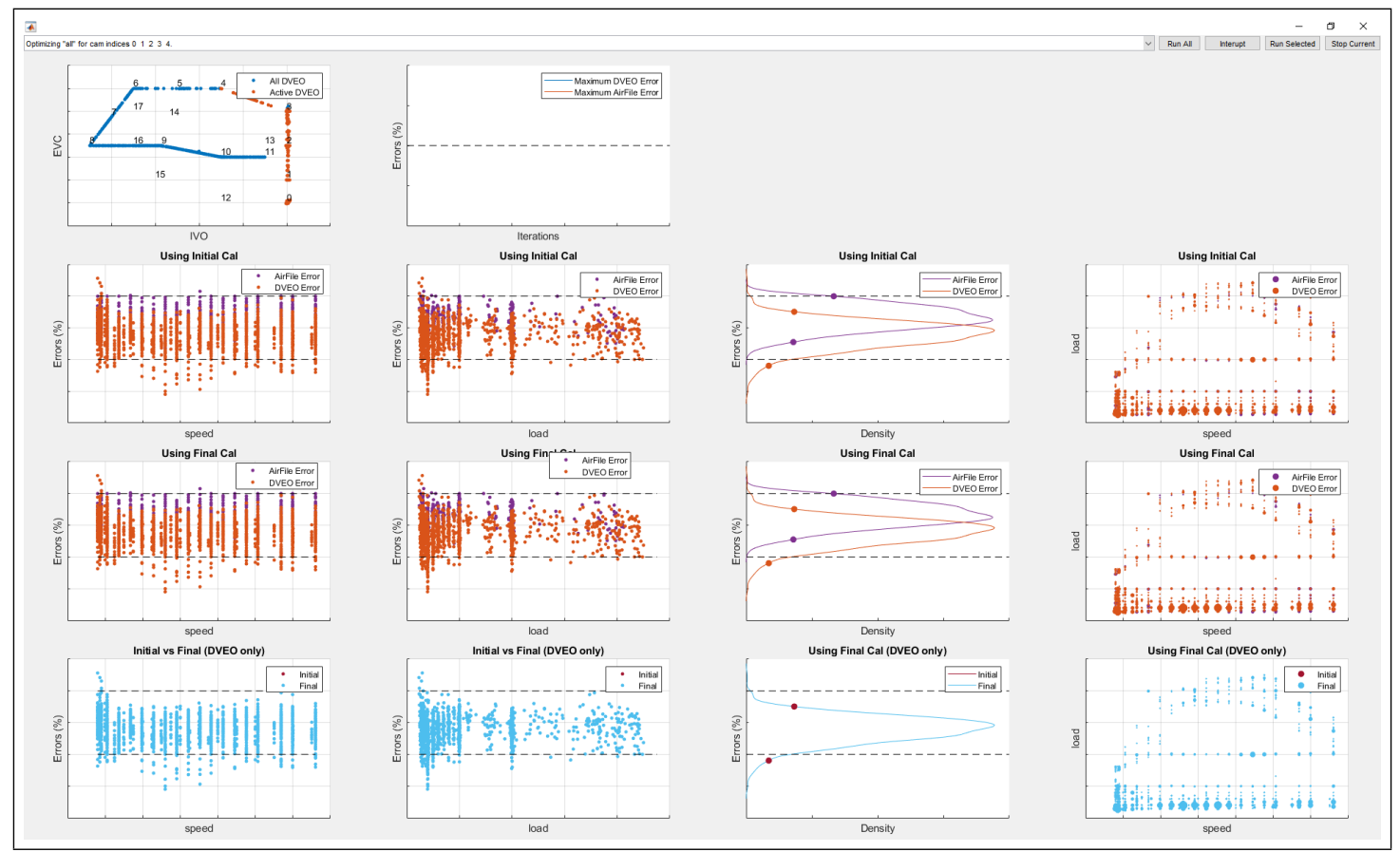

Fig. 5: The optimiser Graphical User Interface (GUI) for test operators which shows the current region of interest, optimisation progress and detailed results in comparison to the Measures of Success (MoS)

checked for modifications. Any test point which will have been affected by the calibration modifications is retested on the dynamometer. This process is repeated until a) all test points are within the MoS, b) the calibration cannot be improved upon by the optimisation routine, or c) the maximum number of experimental iterations has been completed. Subsequently, a report is generated for manual examination by SMEs.

As mentioned in Section IV, the validation process might need to be repeated several times. Like in the manual procedure, the optimisation tool is run after each dynamometer test and outputs not only the updated calibration, but also a minimal set of test points which need to be re-examined. However, unlike the manual procedure, the results do not need to be examined by a calibration SME and the subsequent test can be performed immediately. This dramatically reduces the overall validation time because the engine can be kept running and at normal operating conditions on the dynamometer while the calibration is adjusted and therefore the process can be performed 24 hours a day by test engineers until an acceptable result is obtained. A graphical tool is used to allow engineers to set up the optimisation, run and monitor progress and identify any points where unacceptable errors remain after optimisation, see Figure 5.

\section{CO-Simulation ENVIRONMENT}

Virtual engine testing is a simulation technique which uses a high fidelity engine model developed in speciality software (for example GT Suite [27]-[31], AMEsim [32] or Ricardo WAVE [18]) as a replacement for a real-world engine in order to reduce costs and accelerate calibration development [33]. In order to develop and test the automated process and the optimisation tool, a virtual test bed has been created which connects the MATLAB-based optimisation tool with a high-fidelity Ricardo WaveRT engine-dynamometer plant model and a Simulink-based "ECU".

The co-simulation environment consists of three main components; the WaveRT engine model, the Powertrain Control Module (PCM) or "ECU", and the "Test Scheduler", see Figure 6. The WaveRT engine model is a OD representation of the 1D air-path engine model created in Ricardo Wave by Ricardo PLC [34]. Both the original Wave model and the automatically generated WaveRT model have been correlated to Ford test data by Ricardo PLC. Therefore, for this work, the engine model has been treated 


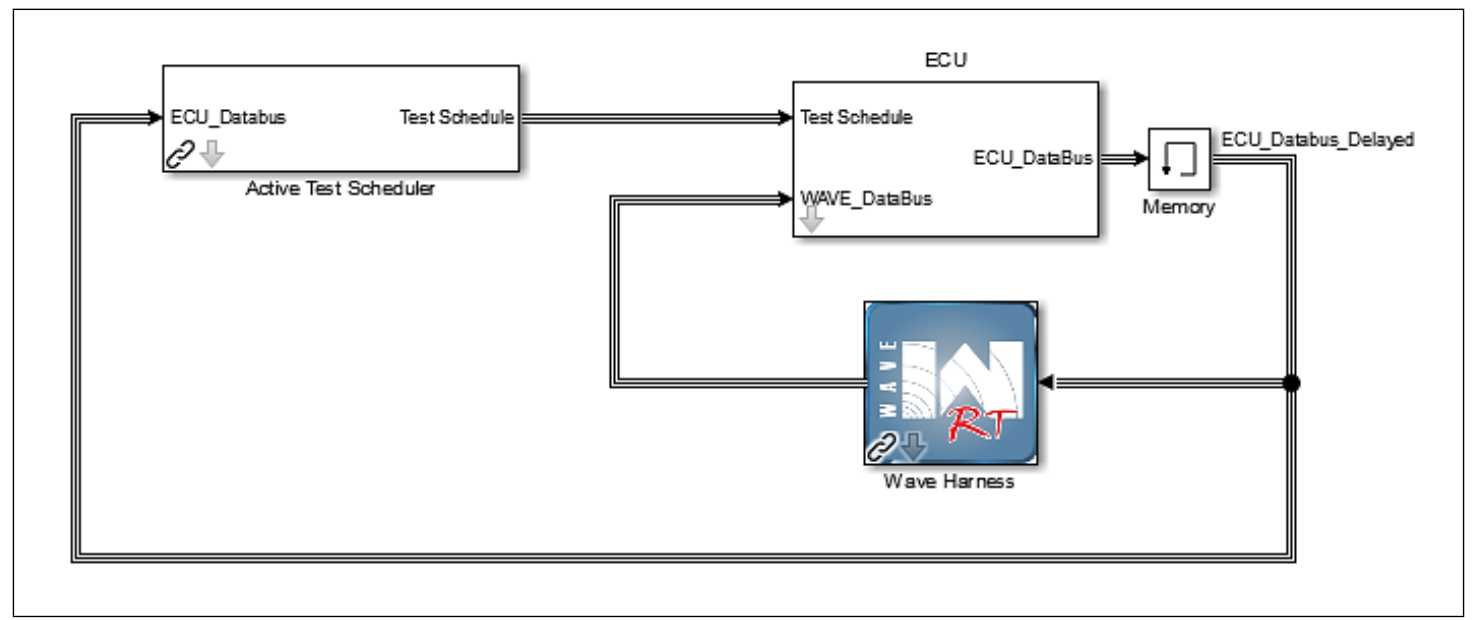

Fig. 6: Co-Simulation Simulink ${ }^{\circledR}$ Model

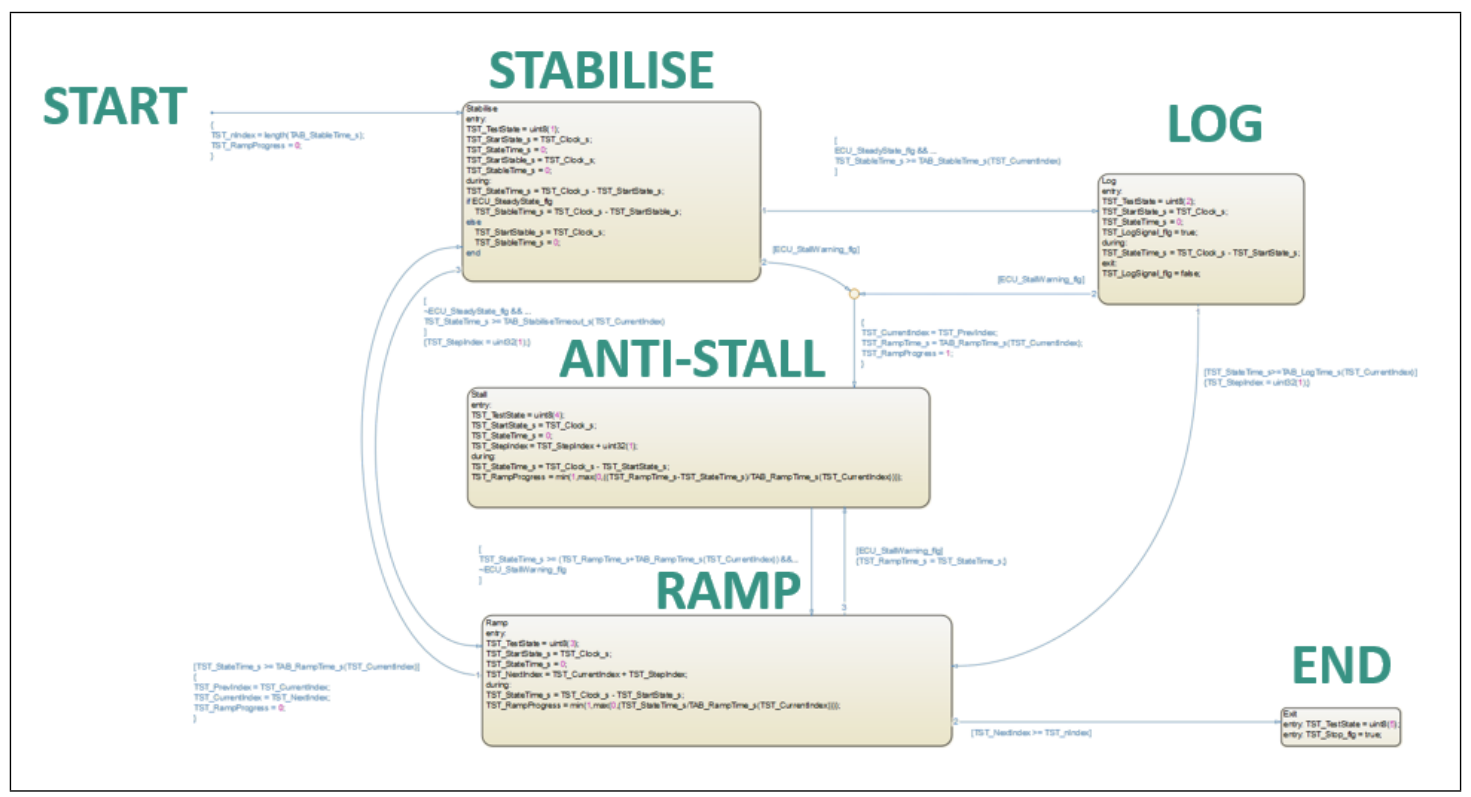

Fig. 7: Test Scheduler StateFlow ${ }^{\circledR}$ Diagram

as a "black-box" model which simply outputs the engine airflow, manifold pressure and torque based on a number of engine control inputs including; throttle position, waste-gate position, camshaft timing, ignition timing, etc..

The "ECU" block is a Simulink ${ }^{\circledR}$ representation of the Ford Powertrain Control Module (PCM) which is parametrised with the calibration to be tested. It takes inputs from the test scheduler in the form of an engine torque demand and uses these in conjunction with feedback from engine model's outputs (virtual "sensors") to manage the engine control inputs.

Finally, the "Test Scheduler" is a StateFlow ${ }^{\circledR}$ model of the engine test procedure as would be performed by test cell technicians, see Figure 7. The test scheduler is provided with a complete list of the required test points in a given order. It ramps to each test point from the previous one, waits for stabilisation of the operating point, and then takes a measurement as the average value over a set period of time or number of engine cycles. It works through each test point until all required test data have been collected and then shuts down the engine model.

Further detailed information about the co-simulation environment is available in separate works by the authors [35]. 

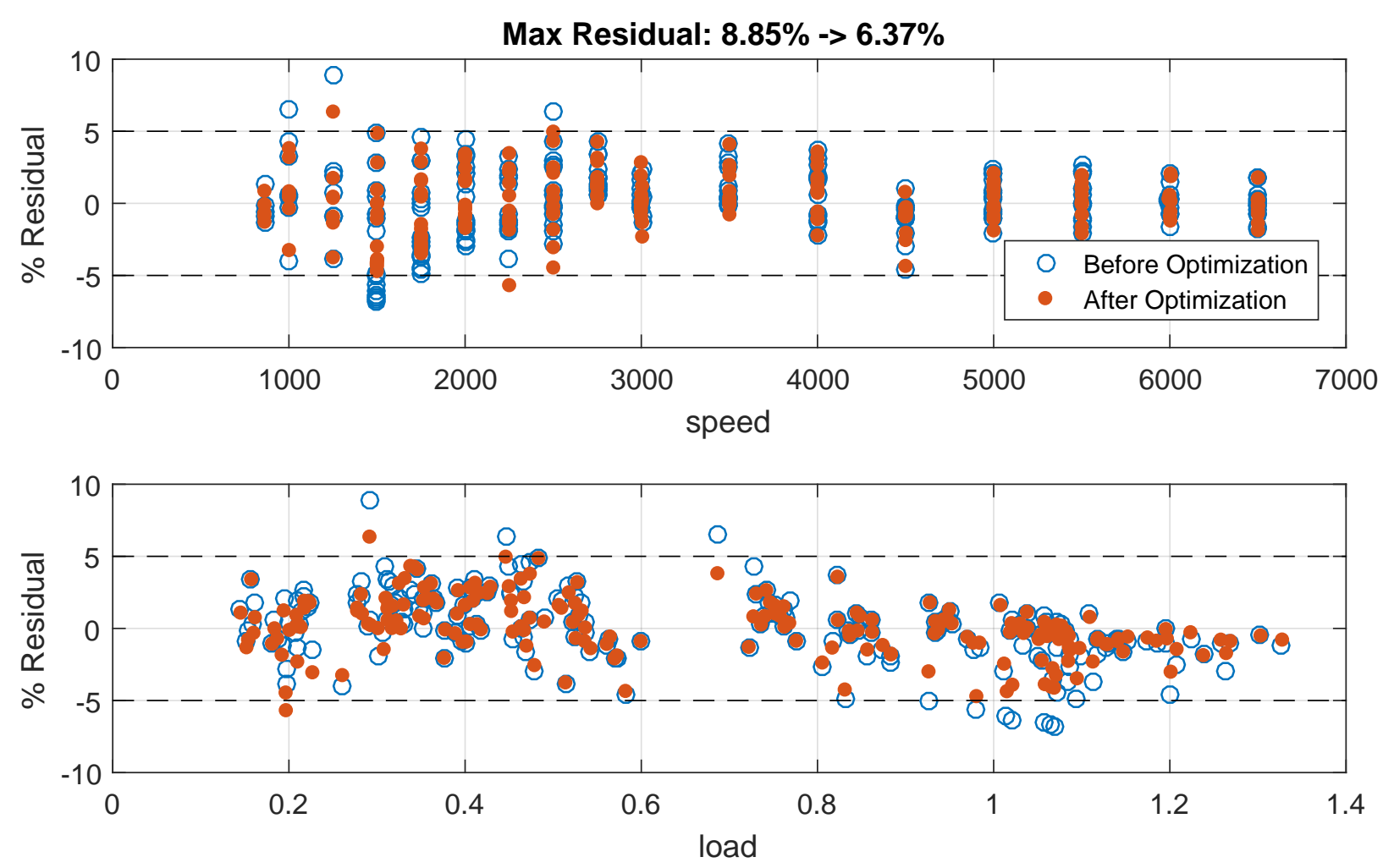

Fig. 8: First Iteration Residuals Before and After Optimisation (Co-Simulation Environment)

\section{Simulation Results And ANALysis}

The iterative process described in Section IV was carried out for a 1.0 litre 3-cylinder Gasoline Turbocharged Direct Injection (GTDI) engine on the co-simulation model. The results of the first iteration are shown in Figure 8 and Figure 9. It can be seen in the blue circles in Figure 8 and in the left plot of Figure 9 that the original calibration did not entirely meet the measures of MoS, which is set to $\pm 5 \%$ relative error. There were around 10 points which exceeded the desired accuracy, with a maximum residual error of $8.85 \%$. In particular, the calibration tended to overestimate at low speed and medium load, but underestimate at low speed, high load. As both of these areas are critical to real-world usage, this calibration would not be acceptable.

The first run of the optimisation function was able to bring all but one of the error states to within the Measures of Success (MoS). However, the optimisation did also push one of the previously acceptable test points outside the desired MoS, meaning that a total of 2 test points were now outside the $5 \%$ relative error tolerance, albeit with a reduced maximum error of $6.37 \%$. In addition to the error states, the first optimisation step also reduced the mean residual slightly.

The change in the calibration will have now affected the actual operating point of a number of test points including, but not limited, to those which were error states under the first dynamometer run and therefore another dynamometer test is performed. The results of the second dynamometer test and the second optimisation run are shown in Figure 10 and Figure 11. It can be seen that the maximum relative error was actually smaller than that predicted at the end of the first optimisation run; at just $5.55 \%$ for the test point which was pushed outside the MoS in optimisation step 1.

Examining the results in detail reveals that the previous worst point $(+6.37 \%$ at $1200 \mathrm{rpm}$ and $\approx 0.3$ load) was in fact within the MoS on the second run of the model. This is not necessarily surprising, because the actual operating point of this test point will have changed significantly due to the substantial change in the calibration in this region as a result of to the optimisation routine trying to minimise this error state. 

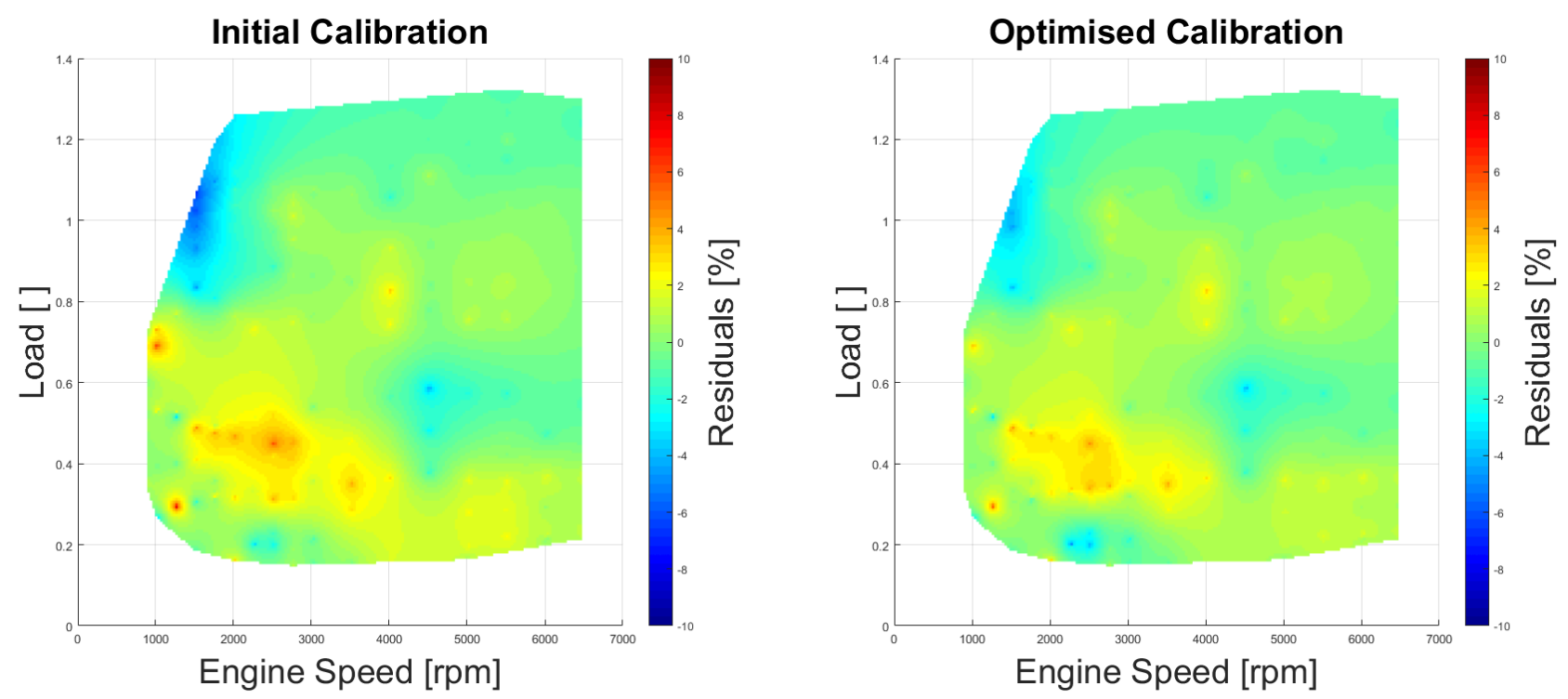

Fig. 9: Map of First Iteration Residuals (Co-Simulation Environment)
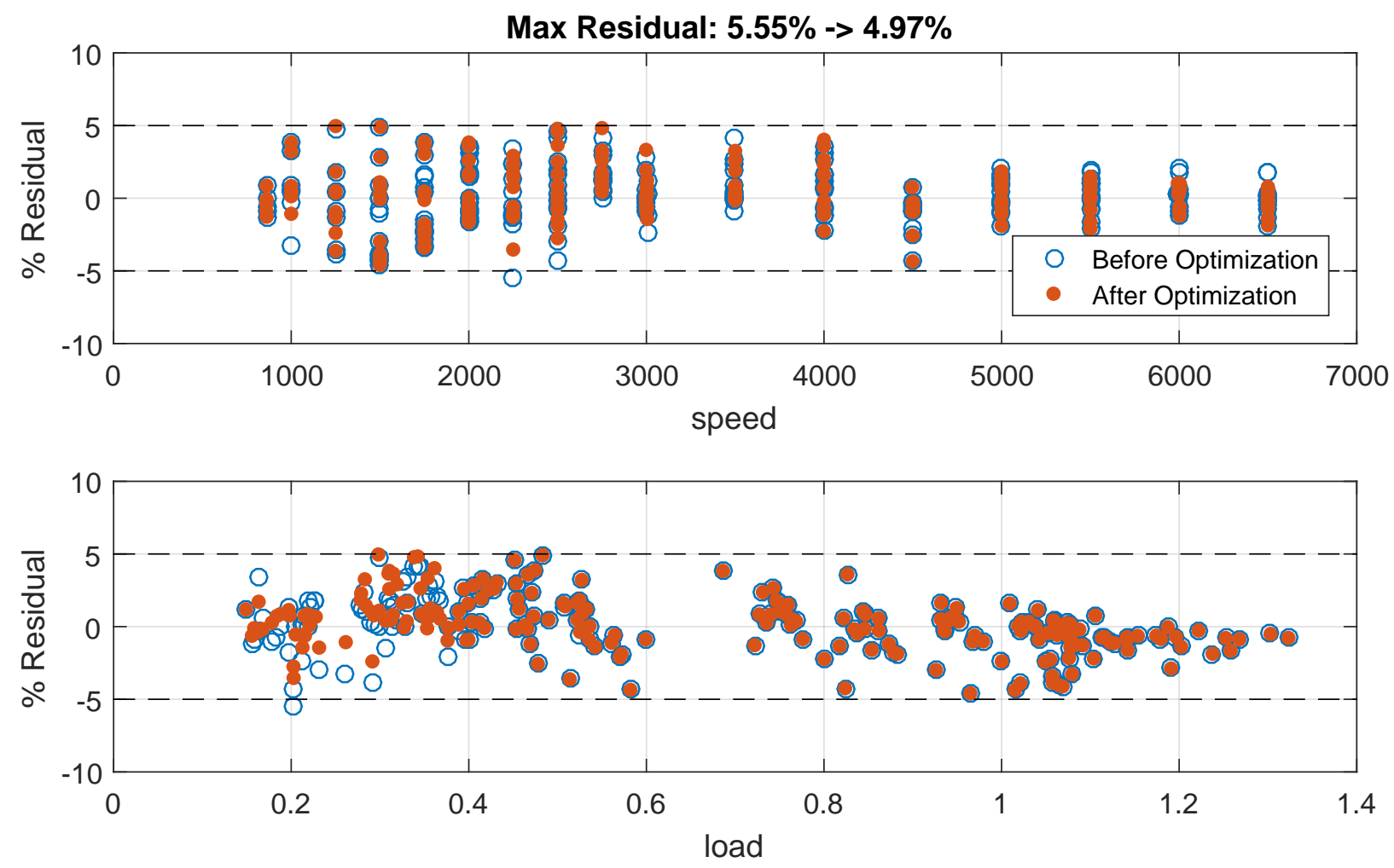

Fig. 10: Second Iteration Residuals Before and After Optimisation (Co-Simulation Environment) 

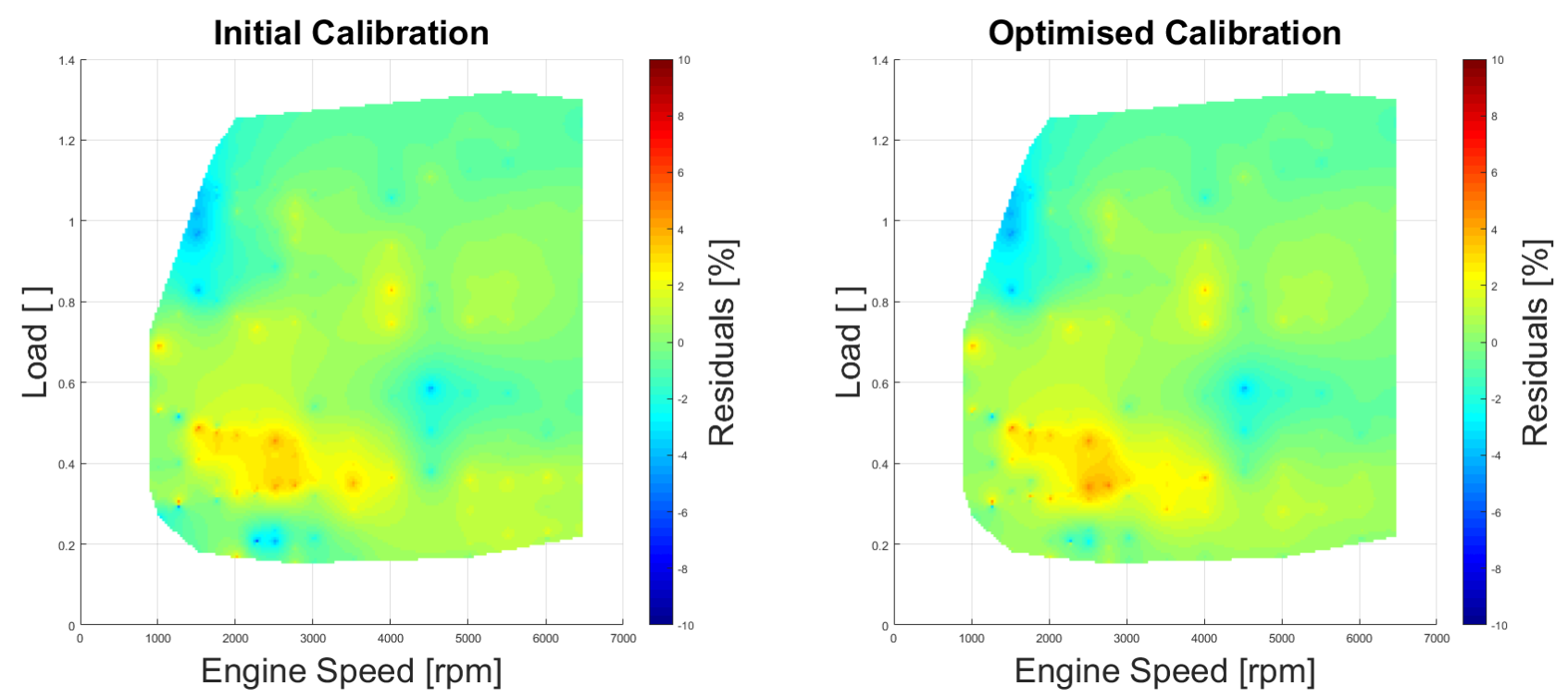

Fig. 11: Map of Second Iteration Residuals (Co-Simulation Environment)

The second iteration was able to bring all test points within the desired MoS with a maximum residual error of $4.97 \%$. However, because this new calibration will have again affected the actual operating point of the engine at each test point, it is still necessary to run the dynamometer co-simulation again. The third co-simulation run showed that the change in calibration had pushed the maximum residual back outside the MoS when accounting for the change in actual engine operating point and therefore the process was iterated twice more until the results of the co-simulation were within the pre-defined MoS. In total, this took a total of 5 simulations and 4 optimisation runs, see Figure 12. Note that the final optimisation run shows no change because it begins by limiting the analysis of the data to test points which are outside the MoS. As there are no longer any error states present in the simulated test data, the optimisation tool reports that the validation process is complete and returns the calibration unchanged.

Figure 13 shows a comparison between the residuals measured in the first validation simulation (Figure 13a, Maximum Residual $=8.85 \%$ ) and the final optimised residuals after all iterations are complete (Figure 13b, Maximum Residual=4.97\%). It can be seen that not only have error states been rectified, but also that the residuals of validated test points have been brought closer to zero. Overall, the Root Mean Squared Error (RMSE) of the test points has been reduced from $2.43 \%$ in the original test run to RMSE $=1.96 \%$ after optimisation.

Each validation test run took around 1.5 hours running on a 4-core parallel simulation, which was equivalent to around 9 hours of experimental testing. The optimisation routine took around 1 hour to complete for each iteration, resulting in a total simulated optimisation time of just under 12 hours. However, simulation is not the end goal of the project and therefore it is useful to consider the total time for the experiment on a dynamometer which would be around 49 hours, representing an approximately $90 \%$ reduction in turnaround time compared to the manual procedure.

\section{PRoduction Deployment}

At the time of writing, the first release version of the optimisation tool has been deployed on five engine programs by Ford UK including multiple air induction and exhaust designs per program. The process is also planned for deployment in Ford North America in 2019. Early results show a typical validation time has been reduced by around 50\% resulting in estimated cost savings in the region of around $\$ 42 \mathrm{~K}$ per programme. In addition to the cost savings, the quality of the calibration has been improved significantly through both reduction of the RMSE by around $50 \%$ and the elimination of systematic offsets. In all 


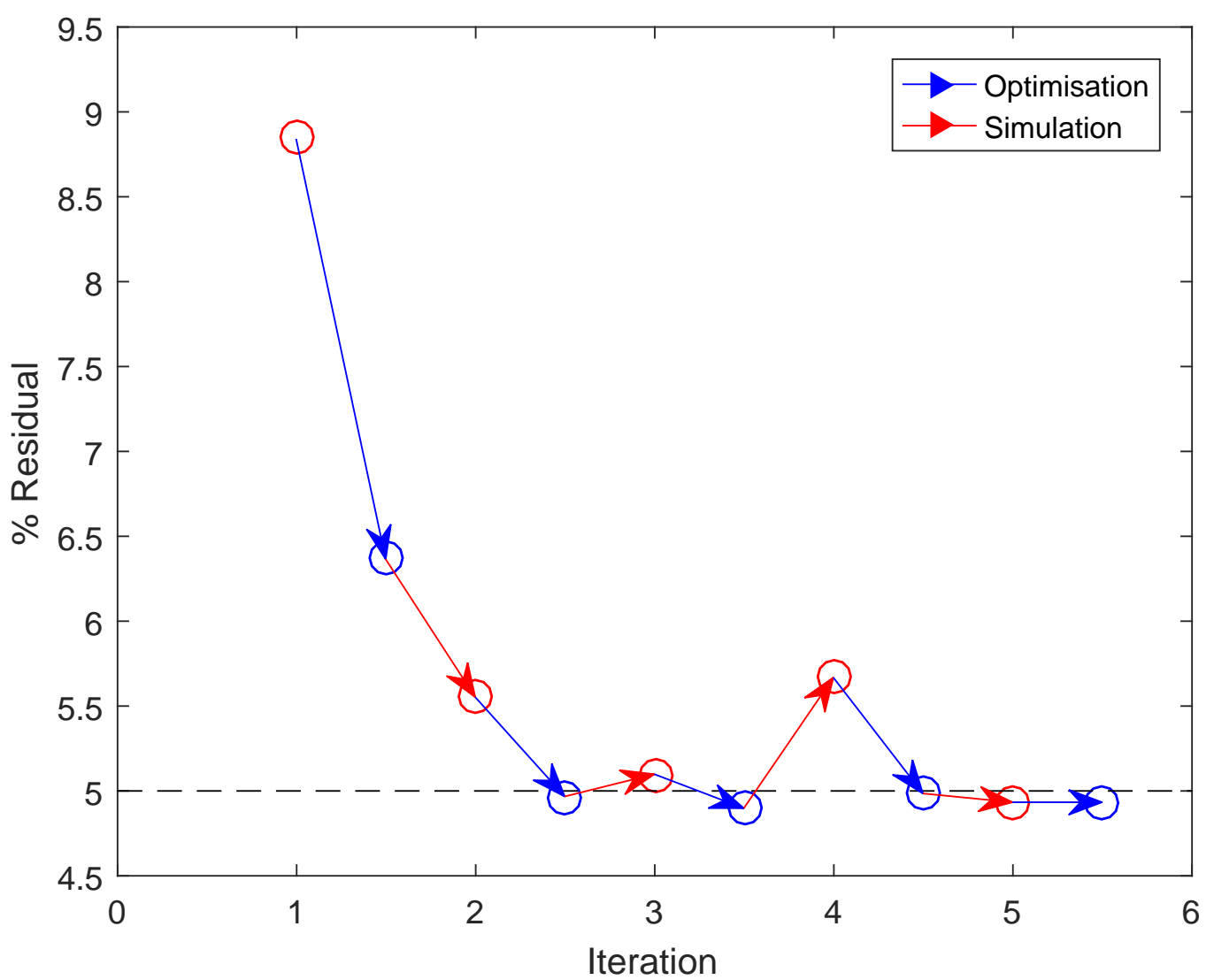

Fig. 12: Maximum Residual by Iteration (Co-Simulation Environment)

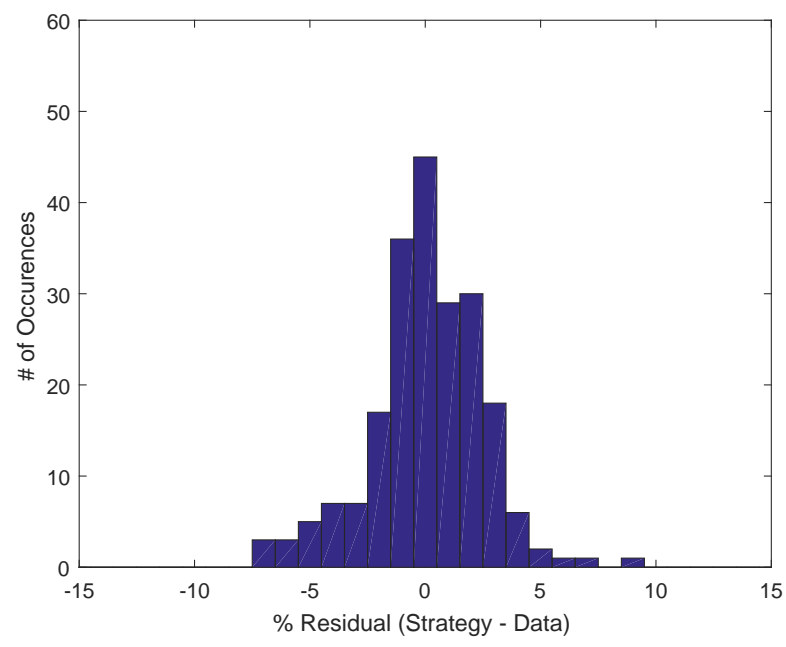

(a) Initial Simulation Residuals: RMSE $=2.43 \%$

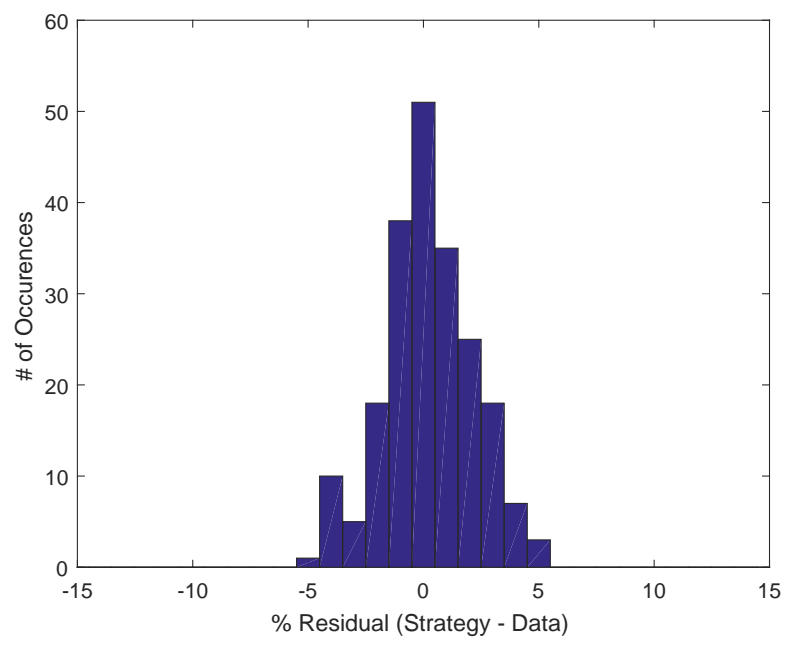

(b) Final Optimised Residuals: RMSE $=1.96 \%$

Fig. 13: Comparison of Simulated Validation Results 
cases, the calibration has met the defined MoS tested to date with more than $95 \%$ of the test points within $\pm 5 \%$.

Figure 14 shows a comparison of the residual air charge errors remaining after manual and automated validation for a single engine configuration. The top plots show histograms of the residual error for each method, and the lower plots show how the error compares to the engine operating point. Comparing Figures $14 \mathrm{a}$ and $14 \mathrm{~b}$, it can be seen that the number of error states outside of the $5 \% \operatorname{MoS}$ has been dramatically reduced from around $11 \%$ to just $2 \%$. In addition, residuals within the MoS have also been brought closer to zero resulting in a reduction in the RMSE of $37 \%$.

Figure 14c shows the systematic error apparent from the manual process. It can be clearly seen that the residual error at low load tends to be skewed to the positive direction, whereas at high load the residual error is skewed negatively. In contrast, this feature has been virtually eliminated in the optimised validation. This improvement is vastly due to sub-routines developed for the automated method designed to bring the initial LUT closer to the final solution before optimisation as mentioned in Section V. This step is beyond the capabilities of a human engineer because it relies on simultaneous examination and adjustment of the complete dataset incorporating thousands of data-points.

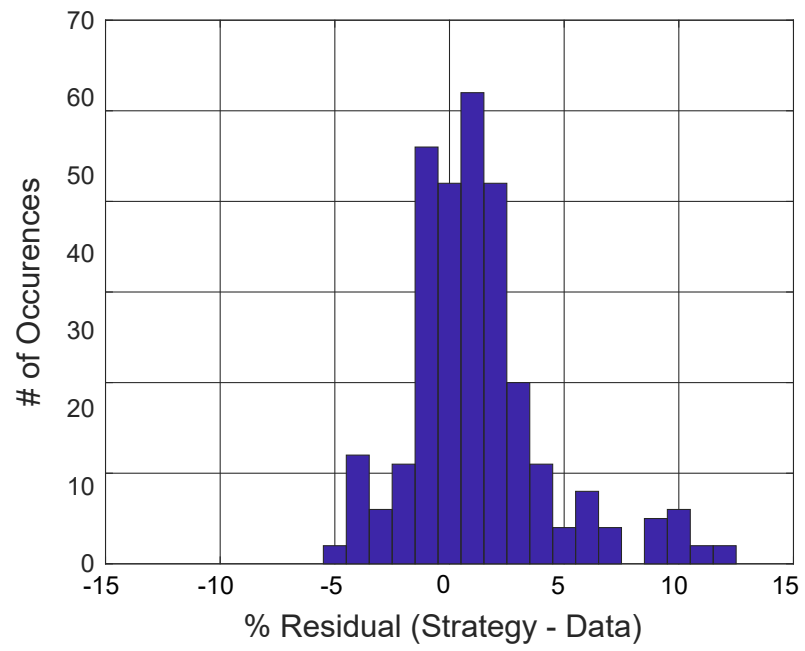

(a) Manual Validation Method: RMSE $=2.28 \%$

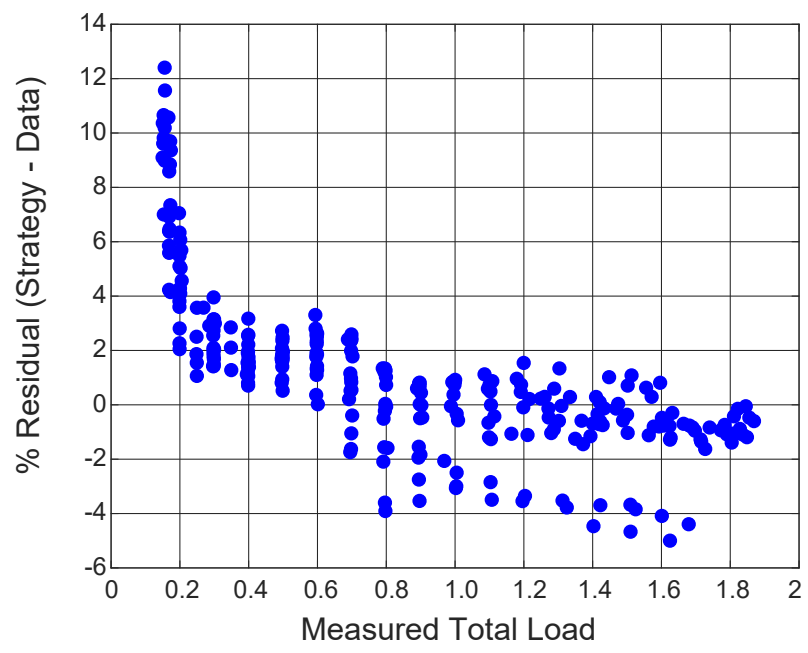

(c) Manual Validation Error vs. Load

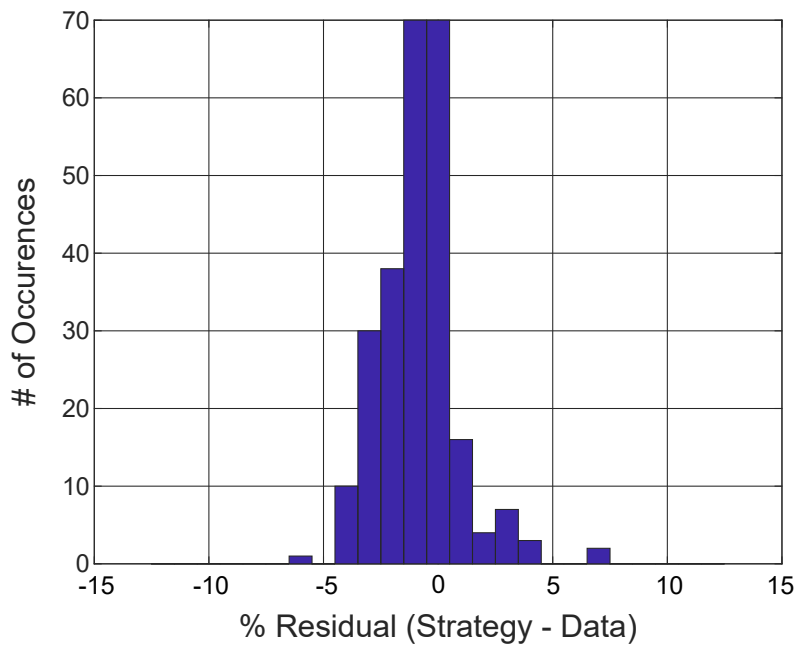

(b) Automated Validation Method: RMSE $=1.43 \%$

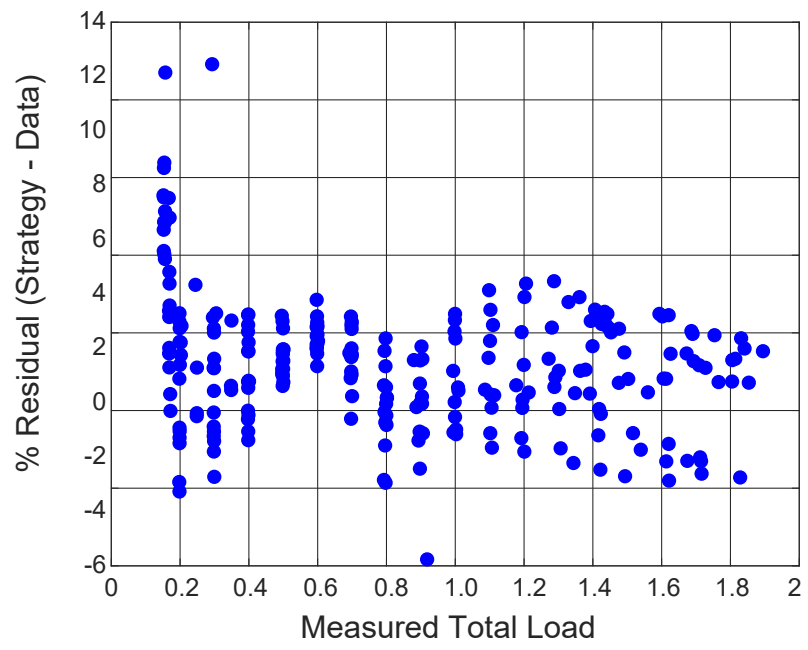

(d) Automated Validation Error vs. Load

Fig. 14: Comparison of experimental validation results. The two plots on the left side show the final experimental residuals after the original, manual optimisation process. The plots on the right show the residuals after the newly developed automated optimisation process. 
Figure 14d shows two test points outside the desired MoS at light loads and a series of test points at very low load which come close to the positive limit. During development of the optimisation routines, it has been identified that these residuals likely result from differences in how the data is collected between the characterisation and validation phases of the calibration process. Consequently, it has not been possible for the optimisation tool to eliminate these error states, however as a direct result of this work, process improvement studies have been initiated at Ford in order to further align the characterisation and validation processes.

\section{CONCLUSIONS}

The simulation results have shown that the proposed method of iteration between co-simulation and optimisation have progressively improved the calibration until it has met the desired MoS. It has also been demonstrated that, for the co-simulation model at least, it has been possible to meet the desired MoS of $\pm 5 \%$ for all test points with a single optimised calibration. It is also interesting to note that, as anecdotally experienced with manual calibration methods, the automated iterative process showed simulated results which were worse than the previous iteration due to the movement of the actual operating point of the engine for a pre-defined test point.

The simulated optimisation demonstrated a theoretical reduction in the calibration validation time from several weeks to just 2 days on an engine dynamometer. This improvement comes from a combination of factors. Firstly, the automated procedure can be run on a 24-hour basis rather than being limited to supervised one or two shift testing. Secondly, the elimination of manual calibration changes significantly reduces the logistical delays of scheduling testing, expert analysis and re-testing as required. Finally, the automated optimiser is significantly quicker than manual examination of the immense volume of test data.

Experimental trialling at Ford has demonstrated that the automated process can be successfully integrated into existing calibration processes. So far, the automated validation optimisation has reduced the validation time of the calibration by around $50 \%$ resulting in estimated cost saving of around $\$ 210 \mathrm{~K}$ across five engine programmes, as well as improving the accuracy of the final calibration by almost $40 \%$ compared to manual processes. The difference between the simulated results and experimental trials can be attributed to additional real-world complexities during testing such as signal noise, environmental factors and experimental errors.

It should be noted however that occasionally automated optimisations need to be interrupted when there is no convergence. These current results mean that the validation optimisation cannot be performed automatically but are able to be quickly performed at the desktop to produce a modified calibration. The optimised calibration can then be tested in the co-simulation environment before resuming testing at the test bench. The modelling environment has been shown to be useful in diagnosing the causes of these interruptions and resolving issues in both the optimisation tool and the calibration process as a whole.

Further work on the VPEC project is continuing at Ford who are working to eliminate the causes of the optimisation failures to maximise the efficiency of the calibration process. In addition, the model has demonstrated substantial correlation to the test data. Therefore, a hybrid simulation/experimental validation method has been proposed to use a combination of simulated results and experimental results to allow the calibration to converge in fewer experimental iterations. 


\section{ACKNOWLEDGEMENT}

This work was completed as part of a collaborative research project, Validation Platform for Engine Calibration (VPEC), organised through the Digital Engineering and Test Centre (DETC), London, UK (www.detc.uk). Project Partners were Ford UK, Loughborough University, Ricardo UK and AVL UK. The authors would also like to thank Ian Noell of Mathworks for his work on the optimisation tool.

\section{ACRONYMS}

$\begin{array}{llll}\text { ADAS } & \text { Advanced Driver-Assistance Systems } & \text { NEDC } & \text { New European Driving Cycle } \\ \text { DETC } & \text { Digital Engineering and Test Centre } & \text { OEM } & \text { Original Equipment Manufacturer } \\ \text { ECT } & \text { Engine Coolant Temperature } & \text { PCM } & \text { Powertrain Control Module } \\ \text { EGR } & \text { Exhaust Gas Recirculation } & \text { RDE } & \text { Real Driving Emissions } \\ \text { FGEC } & \text { Ford Gasoline Engine Control } & \text { RMSE } & \text { Root Mean Squared Error } \\ \text { GTDI } & \text { Gasoline Turbocharged Direct Injection } & \text { SME } & \text { Subject Matter Expert } \\ \text { GUI } & \text { Graphical User Interface } & \text { UIO } & \text { Unknown Input Observers } \\ \text { LUT } & \text { Look Up Table } & \text { VCT } & \text { Variable Cam Timing } \\ \text { MAF } & \text { Mass Air Flow } & \text { VPEC } & \text { Validation Platform for Engine Calibration } \\ \text { MAP } & \text { Manifold Absolute Pressure } & \text { WLTP } & \text { Worldwide Harmonised Light Vehicles Test } \\ \text { MCT } & \text { (Intake) Manifold Charge Temperature } & & \text { Procedure } \\ \text { MoS } & \text { Measures of Success } & & \end{array}$

\section{REFERENCES}

[1] Sangki Park, Youngkun Kim, Seungchul Woo, and Kihyung Lee. Optimization and Calibration Strategy using Design of Experiment for a Diesel Engine. Applied Thermal Engineering, 123:917-928, August 2017.

[2] C. Guardiola, P. Olmeda, B. Pla, and P. Bares. In-cylinder Pressure Based Model for Exhaust Temperature Estimation in Internal Combustion Engines. Applied Thermal Engineering, 115:212-220, March 2017.

[3] Peter Mock, Jörg Kühlwein, Uwe Tietge, Vicente Franco, Anup Bandivadekar, and John German. The WLTP: How a New Test Procedure for Cars Will Affect Fuel Consumption Values in the EU. Technical report, The International Council on Clean Transportation, 2014.

[4] Mehrdad Ehsani, Yimin Gao, Ali Emadi, and Kambiz Ebrahimi. Modern Electric, Hybrid Electric, and Fuel Cell Vehicles. CRC Press, 3rd edition, 2018.

[5] J. Oh, S. B. Choi, Y. J. Chang, and J. S. Eo. Engine Clutch Torque Estimation for Parallel-Type Hybrid Electric Vehicles. International Journal of Automotive Technology, 18(1):125-135, February 2017.

[6] Peyman Niazi, Hamid A. Toliyat, and Abbas Goodarzi. Robust Maximum Torque per Ampere (MTPA) Control of PM-Assisted SynRM for Traction Applications. IEEE Transactions on Vehicular Technology, 56(4):1538-1545, July 2007.

[7] Seta Bogosyan, Metin Gokasan, and Douglas J. Goering. A Novel Model Validation and Estimation Approach for Hybrid Serial Electric Vehicles. IEEE Transactions on Vehicular Technology, 56(4):1485-1497, July 2007.

[8] Seungwuk Moon, Ilki Moon, and Kyongsu Yi. Design, Tuning, and Evaluation of a Full-Range Adaptive Cruise Control System with Collision Avoidance. Control Engineering Practice, 17(4):442-455, April 2009.

[9] Javier Franco, Matthew A. Franchek, and Karolos Grigoriadis. Real-time Brake Torque Estimation for Internal Combustion Engines. Mechanical Systems and Signal Processing, 22(2):338-361, February 2008.

[10] D. Pavković, Joško Deur, and Ilya Kolmanovsky. Adaptive Kalman Filter-Based Load Torque Compensator for Improved SI Engine Idle Speed Control. IEEE Transactions on Control Systems Technology, 17(1):98-110, January 2009.

[11] Jing Na, Anthony Siming Chen, Guido Herrmann, Richard Burke, and Chris Brace. Vehicle Engine Torque Estimation via Unknown Input Observer and Adaptive Parameter Estimation. IEEE Transactions on Vehicular Technology, 67(1):409-422, January 2018.

[12] Thomas Eidenböck, Karl Mayr, Werner Neuhauser, and Peter Staub. The New BMW Six-Cylinder Diesel Engine with Three Turbochargers. MTZ worldwide, 73(10):18-25, October 2012.

[13] Frank Schmitt. Powerful Turbocharging System for Passenger Car Diesel Engines. MTZ worldwide, 75(3):12-19, March 2014.

[14] J.W. Grizzle, J.A. Cook, and W.P. Milam. Improved Cylinder Air Charge Estimation for Transient Air Fuel Ratio Control. In Proceedings of 1994 American Control Conference - ACC '94, volume 2, pages 1568-1573, Baltimore, MD, USA, 1994. IEEE.

[15] Mrdjan Jankovic and Steve W. Magner. Cylinder Air-Charge Estimation for Advanced Intake Valve Operation in Variable Cam Timing Engines. JSAE Review, 22(4):445-452, October 2001.

[16] Ilya V Kolmanovsky and Alexander Anatoljevich Stotsky. Method and System for Engine Air-Charge Estimation, October 2003.

[17] Per Andersson. Air Charge Estimation in Turbocharged Spark Ignition Engines. PhD thesis, Linköping University, Linköping, 2005.

[18] Tom Fletcher, Nikolaos Kalantzis, Mark Cary, Bob Lygoe, Antonios Pezouvanis, and Kambiz Ebrahimi. Automated Model Based Engine Calibration Procedure using Co-Simulation. In 4th Biennial International Conference on Powertrain Modelling and Control (PMC 2018), page 118, Loughborough, 2018. 
[19] Mohammadjavad Ghomashi, Byron Mason, Mark Cary, Kambiz Ebrahimi, and Aitshaam Shahzad. Dynamic Modelling of the Turbocharged Gasoline Direct Injection Air-Path Using Mean Value And Linear Parameter Varying Models. International Journal of Powertrains, 7(1/2/3):15, 2018.

[20] Naoki Amano. Method of Calculating Engine Torque. Patent, 2004.

[21] Qadeer Ahmed and Aamer Iqbal Bhatti. Estimating SI Engine Efficiencies and Parameters in Second-Order Sliding Modes. IEEE Transactions on Industrial Electronics, 58(10):4837-4846, October 2011.

[22] Jonathan Chauvin, Gilles Corde, Philippe Moulin, Michel Castagne, Nicolas Petit, and Pierre Rouchon. Real-Time Combustion Torque Estimation on a Diesel Engine Test Bench Using Time-Varying Kalman Filtering. In 43rd IEEE Conference on Decision and Control, volume 2, pages 1688-1694, Atlantis, Paradise Island, Bahamas, December 2004. IEEE.

[23] Masahiro Yamazaki and Masami Iwase. State Estimation of Internal Combustion Engine Based on Mathematical Model. In V. Duy, T. Dao, I. Zelinka, S. Kim, and T. Phuong, editors, International Conference on Advanced Engineering Theory and Applications, pages 383-393. Springer, Cham, Ho Chi Minh City, 2018.

[24] Alberto Bemporad, Daniele Bernardini, Ruixing Long, and Julian Verdejo. Model Predictive Control of Turbocharged Gasoline Engines for Mass Production. In SAE Technical Paper 2018-01-0875. SAE International, April 2018.

[25] Thomas Harding, Clement Rames, Huang Yu Teh, Toby Mill, Jing Na, Anthony Chen, and Guido Herrmann. Engine Torque Estimation with Integrated Unknown Input Observer and Adaptive Parameter Estimator. IFAC-PapersOnLine, 50(1):11058-11063, July 2017.

[26] Alexander Stotsky and Ilya Kolmanovsky. Application of Input Estimation Techniques to Charge Estimation and Control in Automotive Engines. Control Engineering Practice, 10(12):1371-1383, December 2002.

[27] Charles-Francois Tumelaire, David Gurney, Tony Cains, Nebojsa Milovanovic, and Marco Warth. Flexible ECU Function Development Calibration and Engine Performance Assessment Based on Co-Simulation. In SAE Technical Paper 2013-01-0342. SAE International, April 2013.

[28] Patrick Barasa, Ye Tian, Stefan Hardes, Shahaboddin Owlia, Purvi Limaye, Derek Bailey, and Tarun Sehgal. Virtual Engine, Controls, and Calibration Development in Automated Co-Simulation Environment. In SAE Technical Paper 2016-01-0090. SAE International, April 2016.

[29] Lakshmidhar Reddy Uppalapati, Bruce Vernham, and Yifan Wei. Development and Validation of Engine Calibration Using 1D Predictive Models. In SAE Technical Paper 2019-01-1135, volume 1. SAE International, April 2019.

[30] Tom Fletcher, Nikolaos Kalantzis, Ahmed Ahmedov, Ruoyang Yuan, Kambiz Ebrahimi, Nilabza Dutta, and Christopher Price. Holistic thermal energy modelling for full hybrid electric vehicles (hevs). In SAE Technical Paper 2020-01-0151. SAE International, April 2020.

[31] Nikolaos Kalantzis, Tom Fletcher, Ahmed Ahmedov, Ruoyang Yuan, Antonios Pezouvanis, Kambiz Ebrahimi, Sina Shojeai, and Richard Osborne. Co-simulation methods for holistic vehicle design: A comparison. In SAE Technical Paper 2020-01-1017. SAE International, April 2020.

[32] T. Q. Dinh, J. Marco, D. Greenwood, L Harper, and D Corrochano. Powertrain Modelling and Engine Start Control of Construction Machines. In 3rd Biennial International Conference on Powertrain Modelling and Control (PMC 2016), Loughborough, September 2016.

[33] Ethan Faghani, Jelena Andric, and Jonas Sjoblom. Toward an Effective Virtual Powertrain Calibration System. In SAE Technical Paper 2018-01-0007. SAE International, April 2018.

[34] Robert Norris. Fitting a 1D Gasoline Engine Model at Full and Part Load. In 4th Biennial International Conference on Powertrain Modelling and Control (PMC 2018), page 202, Loughborough, 2018.

[35] Nikolaos Kalantzis, Tom Fletcher, Antonios Pezouvanis, Kambiz Ebrahimi, Mark Cary, and Bob Lygoe. Modelling Environment for Holistic Vehicle Simulation. In 4th Biennial International Conference on Powertrain Modelling and Control (PMC 2018), page 120, Loughborough, 2018. 